\title{
Mapping solution of Interscale Landscape Diversity Modelling Methodology
}

\author{
Györk Fülöp ${ }^{1}$
}

\section{N F O}

Received 26 Nov 2013

Available on-line 31 Dec. 2013

Responsible Editor: Rajkai, K.

Keywords:

Landscape diversity, spatial scales, perception resolution, image information mining, INLAND map

\begin{abstract}
$\underline{\text { A B S T R A C T }}$
Interscale Landscape Diversity Modelling Methodology (INLAND) was set up in order to carry out landscape diversity modelling tasks. The modelling has dual aims: sampling and mapping. In the confines of this paper I introduce the mapping solution, with the description of the modelling needs, and the application of the output results. INLAND methodology defines landscape diversity as the (human/social) perception of spatial heterogeneity. INLAND also intends to model landscape diversity continuously throughout spatial scales. The automated assessment tools of INLAND carry out the modelling tasks relying on Earth Observation (satellite remote sensing) imagery. With the use of sampling tool - objective parameters can be extracted from input data sources. Mapping solution visualises landscape diversity in a map format in order to provide basis for monitoring, and planning tasks - in comfort with the requirements of European Landscape Convention (2000, Florence). Examples of the application of INLAND maps are also introduced along a case study (Rostock region - Mecklenburg-Vorpommern, Germany).
\end{abstract}

\section{Introduction - Challenges of modelling landscape diversity}

Landscapes must be defined, assessed and monitored (European Landscape Convention, 2000, Florence. Assessment and monitoring intentions assume objective description of landscapes' manifestation. One of the most global characteristics of landscapes is their diversity. Landscapes are "a mosaic of heterogeneous land forms, vegetation types, and land uses" (Urban et al., 1987) - heterogeneity is even a defining value of landscapes.

What is the difference between heterogeneity and diversity? Landscape, as a phenomenon, is an "antropo-socio centric" concept (Möcsényi, 1968), which means that our approach to landscapes is naturally considered from the viewpoint of our society. Heterogeneity is a purenatural factor of landscapes, while "the Landscape is a complex entity, developing in the interaction of nature and society" (Csemez, 1996; translation by the author). Heterogeneity must be understood/recognised/felt by the society, to let us speak about landscape diversity. This process of understanding is perception. Perception makes difference between heterogeneity and diversity. The term "Landscape Diverstity" (LD) is used in this paper as: perception of spatial structural heterogeneity.

Spatial heterogeneity, thus landscape diversity is a global value of landscapes. However, landscapes have no specific scale: landscape is a cross-scale concept. Spatial heterogeneity and landscape diversity are also phenomena, which are continuous through all scales. "Spatial heterogeneity is ubiquitous across all scales and forms the fundamental basis of the structure and functioning of landscapes, be they natural or cultural" (Wu, 2004)

According to the definitions above there are two main challenges of modelling landscape diversity: (1) the modelling methodology must be able to describe landscape diversity through spatial scales; (2) and have to model landscape diversity as the perception as spatial

${ }^{1}$ Györk Fülöp

PhD student - Corvinus University of Budapest, Doctoral School of Landscape Architetecture and Landscape Ecology, Department of Biometry and Agricultural Informatics

gyork.fulop@uni-corvinus.hu 
heterogeneity. While spatial heterogeneity is a research focus of landscape ecology, there are no parameters of landscape diversity. While the effect of spatial scale is a research focus of GIS (geographic information systems) and Image Information Mining, there are no operative solutions of data mining to handle scale-continuous landscape analysis.

INLAND (Interscale Landscape Diversity Modelling Methodology) introduces two related automated solutions, which handle "cross-scale" and "perception" problems in an integrated way, when modelling Landscape Diversity according to the input data of Multispectral Earth Observation (satellite remote sensing) imagery. These datasets can provide opportunity for mass-data mining in a homogeneous quality and describe land-cover relations of landscapes.

\section{Data sources - Earth Observation}

Taking landscapes as a perceiver, "landscape is a cultural construction, expressing itself in images, associations and imaginations" (Strohmeier, 2007; translation by the author). If targeting the perceivable aspects of landscapes, it is understandable that from the three above, images are the most objectively accessible sources. The most complex, timely and easily gathered images are the optical Remote Sensing (RS) datasets. The most cost-efficient (on longer terms) way of RS imaging is the satellite based RS: Earth Observation (EO). Assessing the statistical power (unit-defining power) of spatial heterogeneity Nicole et al. also point out: „Satellite imagery is likely to be the only way that such a large volume of data can be collected in a practical fashion" (Nicol et al., 2013).

Earth Observation imagery, if observed from the point of cross-scale priority of LD monitoring, can be classified into three main scales according to the spatial resolution "Overall, more [landscape] metrics showed consistent scaling relations with changing grain size [/spatial resolution] than with changing extent at both the class and landscape levels" (Wu, 2004). Low Resolution (LR) imagery (raster size above $40 \mathrm{~m}$ ) is used for continental, global scale analysis. High Resolution (HR) imagery (raster size 40-5 m) is adequate for regional level information mining. Very High Resolution (VHR) imagery (raster size below $5 \mathrm{~m}$ ) describes local conditions on the Earth surface. These technical scales of input data are discrete, thus investigators are limited to specific scales of observation. While according to the scale dimensions of Dungan et al. (2002) landscape diversity is a continuous phenomena, the possible sampling scales are suffering from discreteness.

\section{Methods}

\subsection{Subscales}

Grain and extent are measures, which are used to describe the spatial scale of an EO image. Grain represents the finest entity in an observation set (spatial resolution, ground size of pixels), while extent is the span of all detected entities (size of acquisition, number of pixels) (Allen et al, 1991). The robust methodology of INLAND intends to give a possible solution for measuring continuous landscape diversity across scales, only with the modification of spatial resolution (degradation of images), without the changing of the extent between the discrete scales.

The concept of INLAND is technically based on the most simple hierarchical (quadtree) (Safavian 1991) balanced image degradation of discrete scaled input EO data, by collapsing the initial image to successively finer resolutions. The quadtree tiling of maps is also a well known coding in cartography. During the looping degradation process an extendable scalogram structure, the structure of subscales is defined $a$ priori. 
The (multispectral) input image with initial extent, grain and spectral resolution, respectively: (E,G,B), is symmetrically segmented into four (two times two) quarter-tiles, and described with the properties of all included grains into tiles. This description means the aggregation of the information content of the initial grains and the segmentation of the initial extent $\left(\mathrm{e}_{1}, \mathrm{~g}_{1}, \mathrm{~B}\right)$. In the next loop, the new segments are tiled up into four again, and described with the properties of all included grains of the initial image. This means again a finer aggregation of the initial grains, and a new, even smaller extent $\left(\mathrm{e}_{2}, \mathrm{~g}_{2}, \mathrm{~B}\right)$ (Figure 1/a). It must be pointed out here, that $g_{2}$ measured to $G$ is better than $g_{1}$ measured to $G$, while $e_{2}$ measured to $E$ is worse, then $e_{1}$ measured to $E$ even though that both $g_{2}=g_{1} / 2$ and $e_{2}=e_{1} / 2$, because technical (EO) development aims of grain and extent are opposites: decreasing grain and increasing extent. The looping is going on, until $\mathrm{e}_{\mathrm{x}}{ }^{*} \mathrm{~g}_{\mathrm{x}}=\mathrm{E}^{*} \mathrm{G}$ ', where $\mathrm{G}^{\prime}$ is the grain (spatial resolution) of an image, which is from a larger discrete scale (e.g. if the first image was an LR EO image, $G^{\prime}$ describes already the grains of a HR image). Then the aggregation process is continued with the use of the larger scale image data input (E,G',B) (Figure 1/a). It must be observed, that between the discrete input scales the extent is not changing. The dataset gained this looping way, simulates a continuous "zooming" option with the tiles, and offers a possibility to combine different discrete scaled data along a symmetric fractal. The tiles, described by $e_{x}$ and $g_{x}$ are the grains of the "sub"-scales, which originate their information content from a larger discrete scaled input data (Figure 1/b).

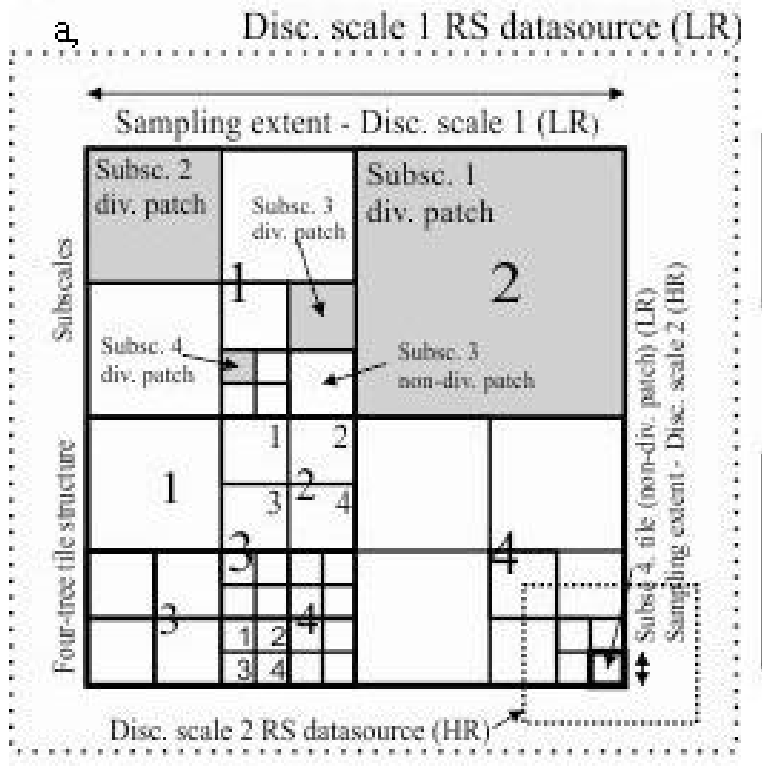

$\mathrm{b}$,

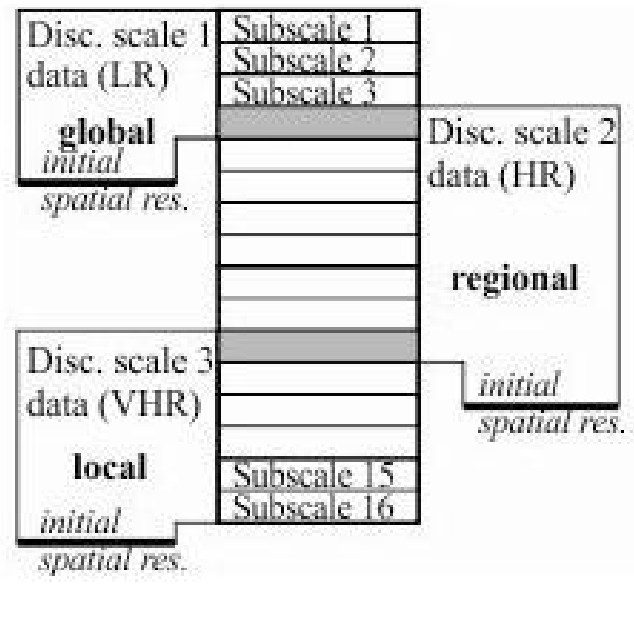

Figure 1. a, Structure of tiling to gain subscale grains; b, Combination of discrete scaled RS data through subscale structure

A similar methodology of image degradation (collapsing the image into successively coarser resolutions) were executed by Woodcock et al. (1987) for selecting the appropriate scale of RS observation to help investigators to choose an appropriate combination of spatial resolution and analysis method. The described process, however, did not include the quadtree aggregation option, causing the pre-definition of degradation scales and the loss of the possibility to combine different discrete scales - the extent of imagery was not assessed).

\subsection{Perception resolution - independent parameter of decision making on heterogeneity}

If the degraded multispectral image tiles of the quadtree based scalogram are conceived to be grains (pixels, rasters) of subscales, decision making process on diversity corresponds with a loop of raster based image classification processes, where diversity patches are defined. Spatial variance, which is the basis of this classification process, is measured in tiles by the 
means and the standard deviation of (spectral) reflectance values of the pixels, which are covered by the tile on the initial input image. The classification leads to a binary decision: across subscales the significantly different tiles $\left(\mathrm{V}_{\mathrm{x}}=1\right)$ are considered to be diversity patches (and not to be tiled into successively finer grains), while the similar ones $\left(\mathrm{V}_{\mathrm{x}}=0\right)$ are proceeded to be reassessed in a larger (with smaller grain size) subscale. This logic is opposite to the well known ECHO image classifier (Landgrebe, 1980), where statistically significantly similar adjacent measurements are classified into aggregate. The INLAND decision making is going on in a loop across every subscale, and uses the following term:

$$
\text { if }\left|A_{y}-a_{y}\right|>=s * d, V_{x}=1 \text {; else } V_{x}=0
$$

,where:

- $V_{x}$ is the output value of the pixels of the initial image, covered by the subscale tile;

- $x$ is the index number of the subscale; $y$ is the index number of spectral Band of the initial image;

- $A_{y}$ is the spectral mean of the input image pixel values of the initial image, which are covered by the containing subscale tile of a smaller subscale (x-1);

- $a_{y}$ is the spectral mean of the input image pixel values of the initial image, which are covered by the containing subscale tile of the assessed subscale (x);

- $s$ is the standard deviation of the input image pixel values of the initial image, which are covered by the containing subscale tile of the decision making subscale (x);

- $d$ is a multiplicator coefficient (later referred as d-coefficient), which is defined by the user, and which modifies the strictness of decision making.

The output elements of this decision making process are the diversity patches indexed with $\mathrm{V}_{\mathrm{x}}$ values in the input image pixels. Diversity patches are the subscale grains (tiles), which differ in their reflectance significantly from the containing grain of a smaller subscale (with coarser resolution). These diversity patches construct a simple scene model during the information mining process. As the decision making is based on the relation of the meandifferences to standard deviation of the tiled pixels, the definition of diversity patches is independent from the global variance of the image. Thus the extraction of diversity patches is also assured from all subscales of combined discrete scale images. Here it must be outlined, that diversity patches are not the outputs of INLAND methodology, they are only used for extracting spatial statistics (sampling tool), and categorical maps of LD (mapping solution).

"The criteria for defining a patch may be somewhat arbitrary, depending on how much variation will be allowed within a patch" (Gustafson 1998), or how much is required to define a new patch. " $d$ " value (d-coeff) in the expression used for binary decision during the diversity patch definition influences and controls directly how much variation is required to define a diversity patch. The d-coeff is setting the rigidity or strictness of the decision making. In this manner it represents the perceivers ability to understand the landscape - or to observe spatial differences. The d-coefficient is measuring the perception resolution, which distinguishes "Dakota Indians" (high perception resolution, small d-coeff values), who recognize even very small differences in the landscape, from "Uptown Infants" (low perception resolution, high dcoeff values), who only think in city-village relations.

As INLAND methodology targets the perceivable spatial heterogeneity (LD), the modelling concept is based on the analysis of the diversity patch structure through subscales in the function of d-coeff (or perception resolution). 


\section{Mapping solution - keeping the model in raster domain}

The sampling solution (published separately - Fülöp et al, 2013) of Interscale Landscape Diversity (INLAND) methodology was set up to measure spatial heterogeneity (define and count diversity patches) in the function of the perception resolution across the subscales of a given discrete scaled image, which is collected from a landscape. This intention assumes, that the scene and accordingly the image) is taken from inside of the boundaries of the functional region of the landscape, which is targeted to be characterized with its LD: there are no landscape boundaries represented on the image. The appearance of such functional landscape boundary was presented with the use of entropy values extracted from polarimetric SAR data on the administrative border of Hungary and Serbia (Fülöp, 2012)

The mapping solution intends to create multi-channelled categorical maps with the use of the methods (subscales, and perception resolution) described above and integrated into the sampling tool. Different channels of the created maps represent different strictness in the decision making process (d-coefficient): the user can observe LD in the function of perception resolution.

As Gustafson (1998) states: "Raster maps represent boundaries as the interface between cells of different classes [diversity patches or non-diversity patches of sampling solution] so that boundaries must conform to the underlying lattice structure. This can have marked effects on the [vector based] delineation of patches." That is also a reason why diversity patches could have not been used directly for mapping purposes: to keep patch delineation in raster domain. Two other, more important but linked causes were (1) the sampling solution's sensibility for functional landscape borders and (2) the inefficient measurement of neighborhood relations (with the quadtree structure the mean of a given subscale tile is related only to three out of eight neighboring tiles during the decision making comparison of the means). These problems all together mean the sampling solutions exposure to the spatial movement of the sampling image extent.

The INLAND mapping solution is based on a repeated sampling structure, which uses the sampling image extent as a moving window, passed over the landscape scene (Figure 2/a). However, because of the subscale structure (which consists of successively finer subscale tiles) the moving degree of the sampling window had to be spatially considered. While tiles from the first subscale (grain: 64 initial pixels) should be compared to the surrounding tiles which have the same tile size: resulting the movement of the sampling window by 64 initial pixels, tiles from the sixth subscale (grain: 2 initial pixels) require the movement of the sampling window only with 2 initial pixels (subscales and window movement degree on Figure 2/a, b and c). 


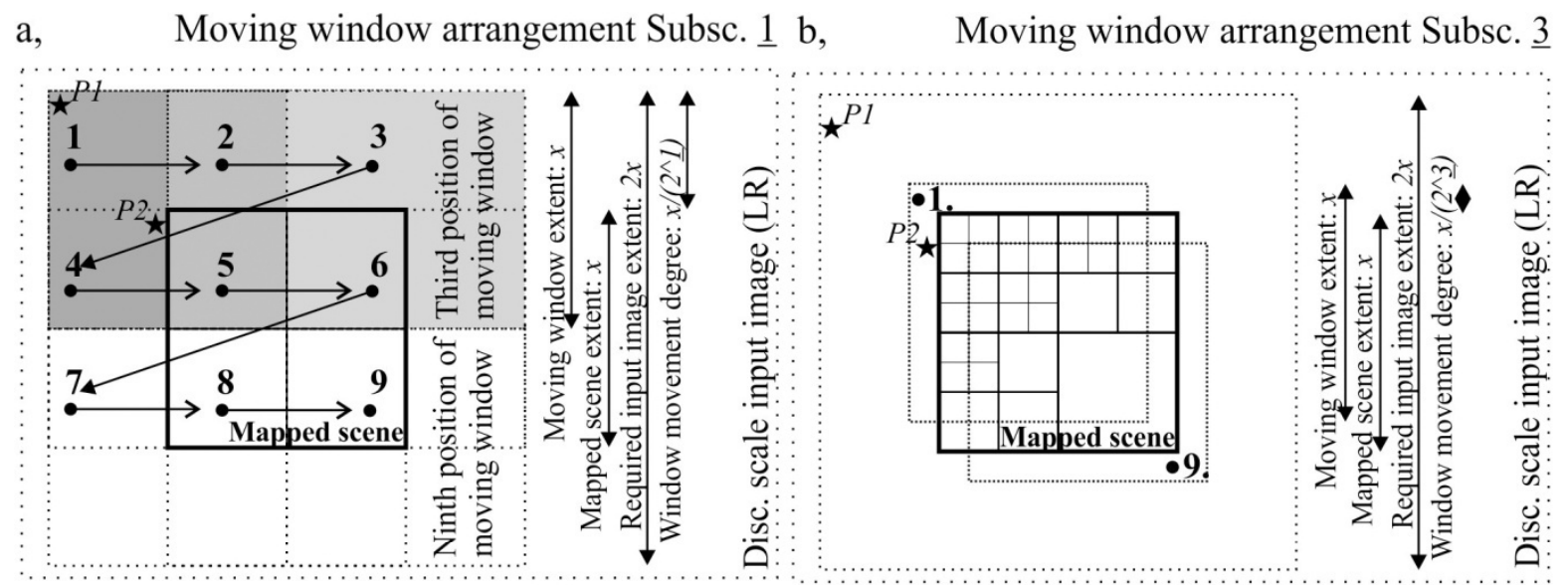

c, Tile comparison with neighbors Subsc. $\underline{2}$

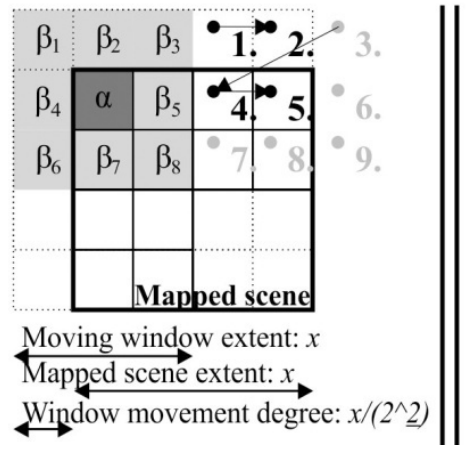

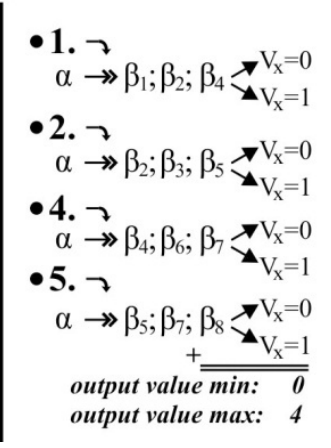

Figure 2. a, Moving window arrangement in subscale 1; b, Moving window arrangement in subscale 3 ; c, Moving window arrangement in subscale 2 - tile comparison with neighbours - extraction of grain value $[0,4]$.

Therefore the repeated sampling with the moving window was integrated into the quadtree structured decision making process to create categorical maps. While the sampling solution consisted of two different looping processes (looping across subscales and looping across perception resolution) the mapping tool integrates between these two a third one: looping across spatially moved windows. The moving window technique is based on a sampling methodology, which also has a spatial extent. In the case of INLAND mapping solution the sampling image extent is the same as the mapping image extent (128), however the analysis assumes the accessibility of double image extent (256) (Figure 2/a and b).

During the extraction of the INLAND map the MATLAB code runs the sampling method at each d-value and each subscale nine times (Figure 2/a, b and c). Each sampling represents a different state of the moving window, assuring that tiles are compared to all of their eight direct neighbors (Figure 2/c). The movement degree of the tile is the function of the assessed subscale (subscales and window movement degree on Figure 2/a, b and c). At the first subscale this movement measures 64 pixels of the initial image, on the second this value is 32 , on the third it is 16 etc. From these nine measurements each assessed tiles participate in four (Figure 2/c). E.g. the first indexed tiles in the first, second, fourth and fifth move of the window, the second indexed tiles in the second, third, fifth and sixth, the third indexed tiles in the fourth, fifth seventh and eighth, the fourth indexed tiles in the fifth, sixth, eighth and ninth movement of the window. Thus each tile can be considered at maximum four times to be a diversity patch at each subscale and perception resolution. In this decision the northern, eastern southern and western neighboring tiles take part twice, while the north-eastern, south-eastern, south-western and north-western tiles only once (Figure 2/c). This weighing represents the edge effect of the tiles originating from the quadtree delineation of the modelled tiles - the last subset of the four neighboring tiles is not connected with the assessed tile along a border line. As the channels of 
the output categorical map represent different perception resolution, at each channel the maximum value of the tiles can be four, while the minimum value zero (defining non-diversity patches). As a result of mapping method each tile ( $\alpha$ on Figure $2 / \mathrm{c}$ ) at every d-value and subscale is measured to its neighboring tiles ( $\beta$ on Figure $2 / \mathrm{c}$ ), and also that is why the mapping solution requires the accessibility of a double image extent as the mapped scene (Figure $2 / a$ ). Because of future research needs (see at Outlook), during the mapping process only the first five subscales were used (the output map has four times bigger grain size than the input image).

Viewing globally the whole mapped scene, the categorical map output describes the evenness (composition index) of diversity patches in the function of perception resolution. Evenness refers to the distribution of area among the classes (diversity patches or non-diversity patches). In the study of Riitters at al. (1995) composition indices (such as evenness) have been also spatially referenced, by calculating the index within a moving window that is passed across the map. Galante et al. (2009) also presents IIM use of moving window structure.

The moving window construction was needed because of the rigid neighborhood-handle of quadtree decision making, also not reflecting spatial construction efficiently right out of the borders of the sampling tool (P1 and P2 on Figure 2/a and b). The altering moving degree of the sampling window assumes that closer pixels have greater influence on perceivable LD (differences can be observed more easily between areas, which are more close to each other): the existence of autocorrelation. This can be easily understood when realizing that the initial image pixel just right out of the boundaries of mapping extent influences diversity patch definition at each subscale (P2 on Figure 2/a and b), while an image pixel 64 pixels remote from the mapping extent has impact only at one subscale: at the first one (P1 on Figure 2/a and b). Autocorrelation means in a simple definition: points closer together tend to be more similar than points farther apart (Gustafson, 1998). Thus the natural spatial similarity (decreased differences) of initial image pixels is represented in the INLAND mapping process.

\section{INLAND map-products and examples of the map-application}

With the use of INLAND mapping solution the landscape diversity maps of MecklenburgVorpommern (MV) (Germany) and Hungary were created. In the confines of this paper the LD map-model of MV, the map-products and the application of these products are introduced in order to illustrate practically the set up of the mapping solution. The Hungarian map will be published when regionally related applied assessments (overlay with the national control plan) are ready.

During the creation of the LD map of MV 32 perception resolution (d-coeff; from 0.1 to 3.6) values were used - thus the map-model consists of 32 Bands - each Band standing for a perception-strength of the perceiver. The total set of these Bands (and the tendencies between them) describe the LD in this region (Figure 3). The resolution of input imagery was $463 \mathrm{~m}$ (MODIS), the output resolution of the model is $1852 \mathrm{~m}$ (four times bigger). The mapping window-extent was 128 (when shifting 256), thus it is clear, that five subscales were used during the mapping process $\left(128 /\left(2^{\wedge} 5\right)=4\right)$. With this mapping extent whole territory of MV was mapped in 17 tiles. 


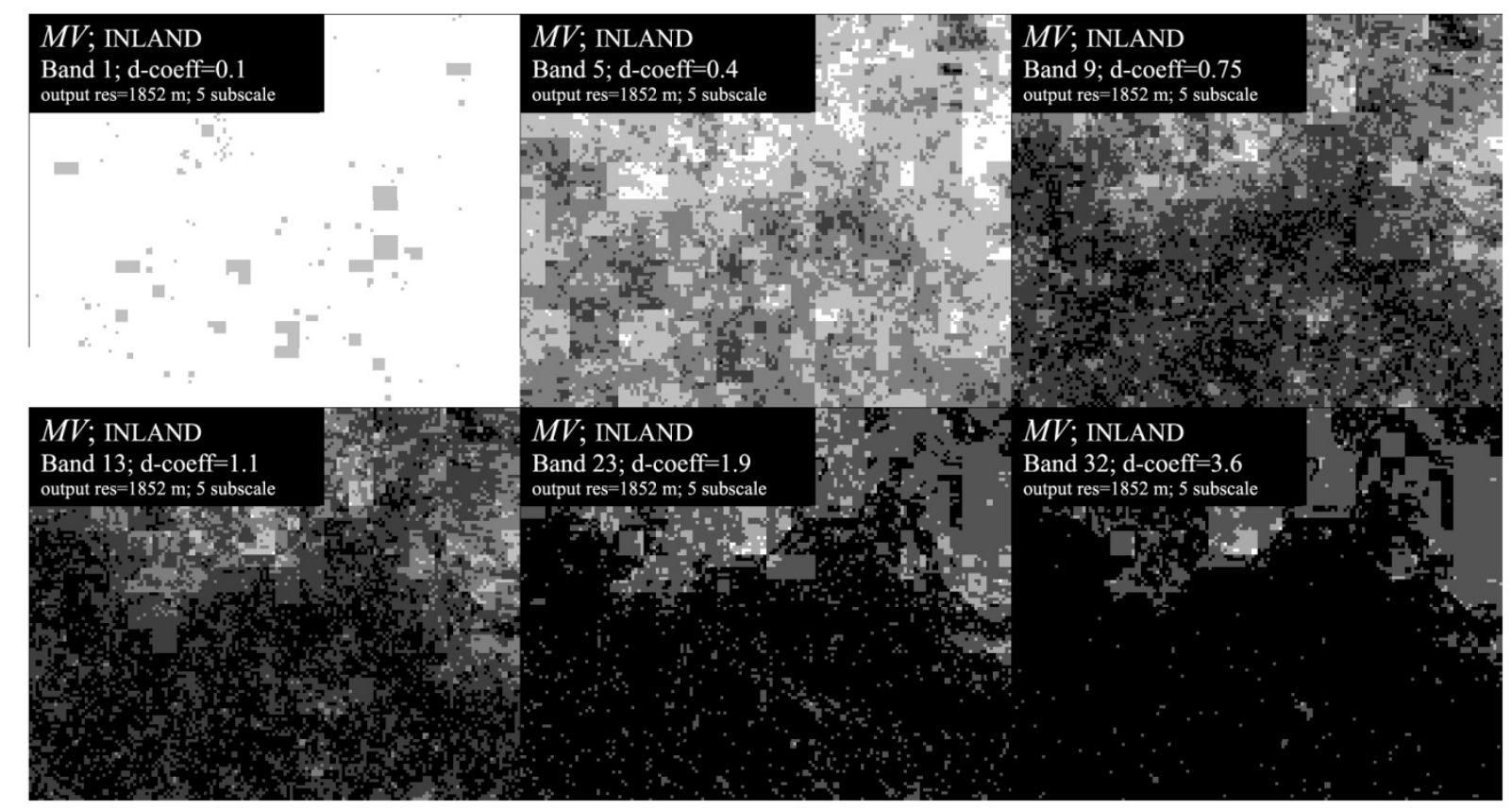

Figure 3. Bands of INLAND map-model of Mecklenburg-Vorpommern (Germany) from 17 mapped scene-tiles (each 128 by 128 pixels). Map-model consists of 32 Bands $\sim 32$ perception resolution value

(d-coeff.) $[0.1,3.6]$; 5-subscales were used, resampled from MODIS imagery (463 m); output resolution is $1852 \mathrm{~m}$; pixel values [0,4] - 0 (black) standing for homogeneity, 1 (white) for heterogeneity.

However, the whole set of 32 Bands is very difficult to understand. In order to interpret this map-model, two types of map-products have been created. The first map-product (Figure 4/a) is a grey-scaled image, indicating the d-coeff. threshold, at which value the part of the landscape is turning from diverse to homogeneous (as the perception resolution becomes coarser). The other map-product is an RGB image, each Band (red, green, blue) standing for a perception group (red is the "expert" perception group - d-coeff. values from 0.1 to 0.9 averaged; green is the "medium" perception group - d-coeff. values from 1 to 2.2 values averaged; while blue is the "weak" perception group - d-coeff. values from 2.4 to 3.6 values averaged) (Figure 4/b).

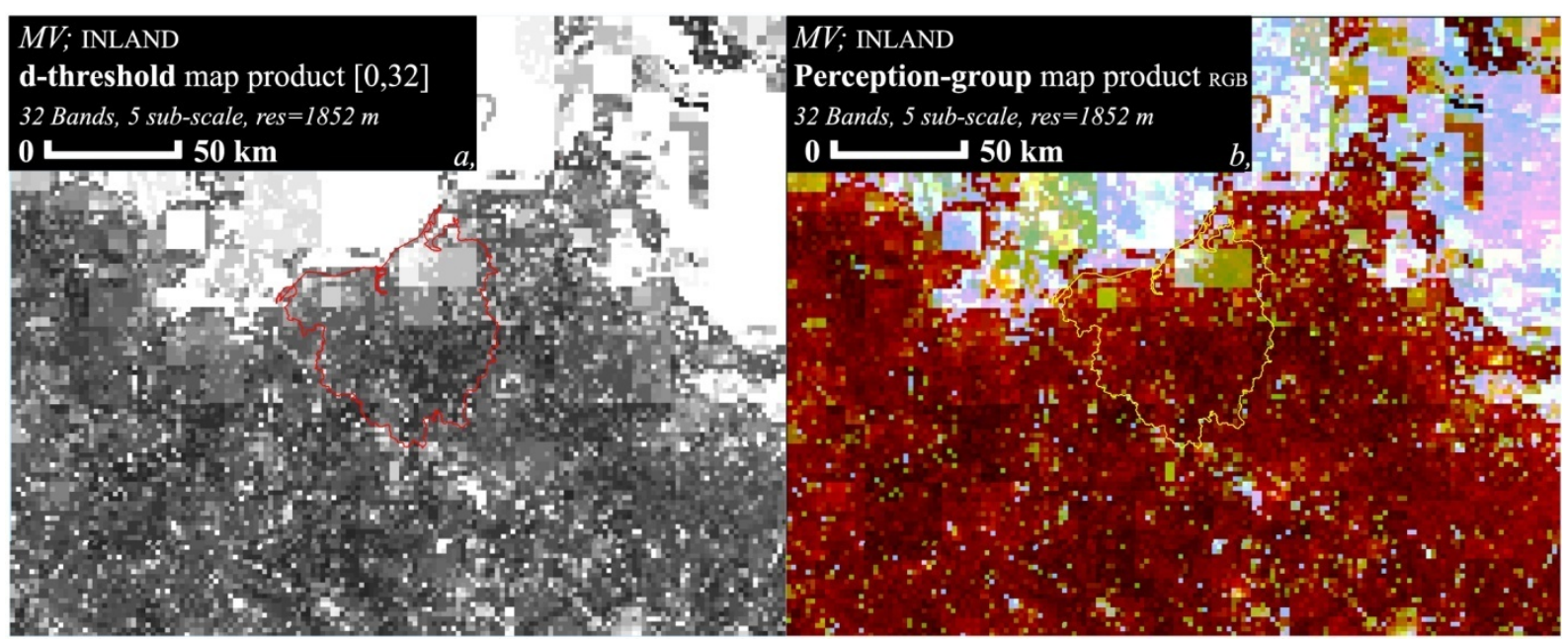

Figure 4. a, d-threshold map-product of INLAND map-model of Mecklenburg-Vorpommern and Rostock Region, pixel values [0,32] standing for by which d-coeff. value area becomes from heterogeneous to homogenous; $b$, perception-group map-product $-\mathrm{R} \sim$ landscape expert $\mathrm{d}=[0.1,0.9]$, $\mathrm{G} \sim$ medium perceiver $\mathrm{d}=[1,2.2], \mathrm{B} \sim$ weak/children $\mathrm{d}=[2.4,3.6]$. 
It must be emphasized, that these maps consider landscape diversity to be a value of its own - not assigning values to different land covers. These map-products were used to analyse the LD related to different land use units, defined in the control-plan of Rostock Region. During the analysis the vector data of the control-plan was overlaid on the raster data of LD mapproducts. As a result of this analysis, a "regional landscape diversity presentation plan" was prepared, which assigns proposals to control-units of the regional control plan. This presentation plan describes, how the (ecologic, aesthetic, economic) values of landscape diversity could be presented for people, who perceive the landscape. As an example, on Figure 5, the LD assessment of nature-protection areas can be seen, according to the d-threshold values. The colour of the areas shows, how much they can serve the aim to present the value of landscape diversity in nature protection areas. The darker the areas are, the more adequate they are for the demonstration of landscape diversity - since even for visitors with lower perceptionresolution the spatial heterogeneity is understandable, recognizable - perceptible.

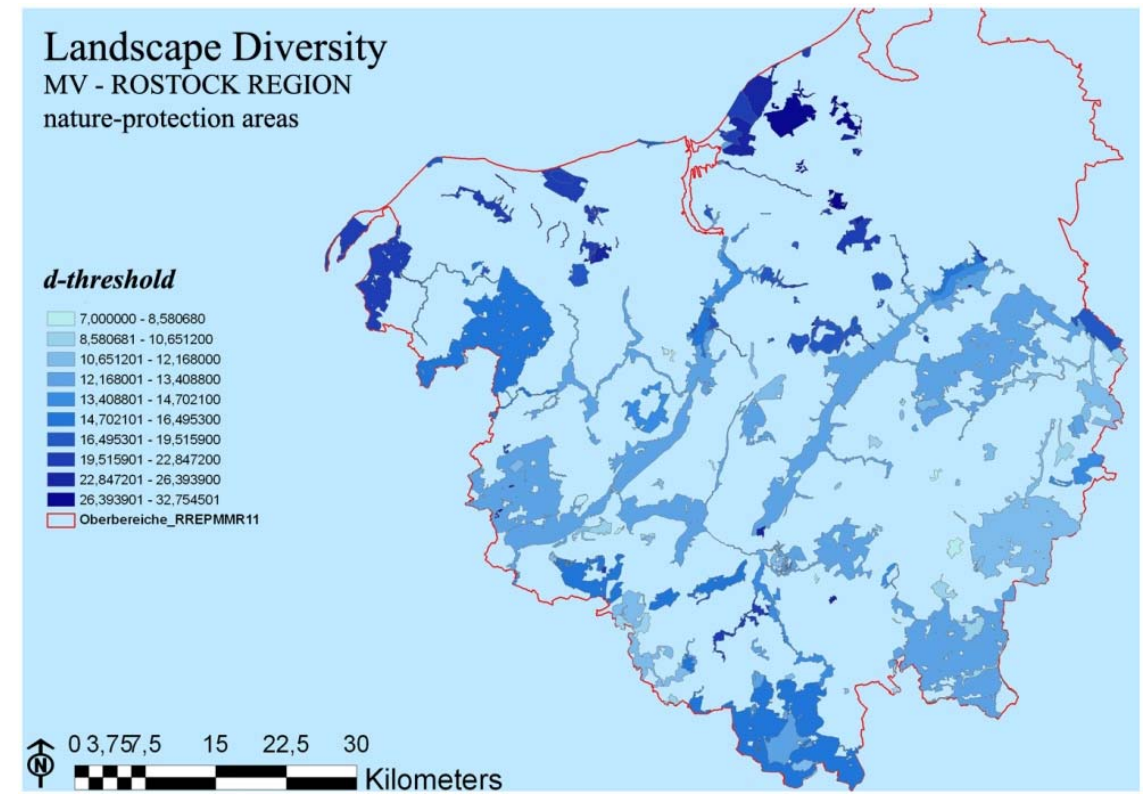

Figure 5. Landscape diversity assessment of nature protection areas in Rostock Region (MV, Germany), with the use of d-threshold map-product of INLAND map-model. Dark blue areas have higher d-threshold, thus in the area LD can be presented for wider group of visitors.

\section{Summary}

INLAND solutions can model landscape diversity continuously across spatial scales, and in the function of human perception. The automated solutions provide opportunity to utilize Earth Observation imagery, thus besides information mining, the way of data collection can also support the operative monitoring of landscape diversity. As a personal opinion: the greatest value of Hungarian landscapes is their diversity. INLAND provides solutions to take diversity as our national value in order to preserve it and to develop it.

\section{Acknowledgements}

The research of INLAND solutions was supported by Deutsche Bundesstiftung Umwelt Scholarship. I thank the thoughtful consultations for Barabásné Dr. Martos Júlia, for Prof. Holger Behm, for Dr. Ladányi Márta and Torma Péter, and the caring advices for two anonym reviewers! 


\section{References}

Allen, T. F. H., Hoekstra, T. W. (1991). Role of heterogeneity in scaling of ecological systems under analysis. Ecological Studies 86: Ecological Heterogeneity, J. Kolasa, and S.T.A. Pickett, eds, Springer-Verlag, 47-68

Csemez, A (1996). Tájtervezés-tájrendezés, Mezőgazda Kiadó, Budapest, ISBN 963736256 8, 278

Dungan, J. L., Perry, J. N., Dale, M. R. T., Legendre, P., Citron-Pousty, S., Fortin, M. J., Jakomulska, A., Miriti, M., Rosenberg, M. S. (2002). A balanced view of scale in spatial statistical analysis. Ecography 25: 626-640

Fülöp, Gy. (2012) IIM intentions to measure landscape diversity. Proc. ESA-EUSC-JRC 8th Conference on Image Information Mining. 24-26.10.2012. Oberpfaffenhofen 86-90 doi:10.2788/49465

Fülöp, Gy. Behm, H. (2013) Interscale Landscape Diversity Modelling Methodology (INLAND) - Mining perceivable landscape diversity information with spatial statistic methods from Earth Observation images Journal of Land Use Scienece - paper under publication

Galante, G., Mandrone, S., Funaro, M., Cotroneo, R., Panetta, S. (2009). Spatial and temporal changes in Aniene river basin (Latium,Italy) using landscape metrics and moving window technique. Italian Journal of Remote Sensing 41(2), 157-172

Gustafson, E. J. (1998). Quantifying Landscape Spatial Pattern: What Is the State of the Art? Ecosystems (1), $143-156$

Landgrebe, D. A. (1980). The development of a spectral-spatial classifier for earth observation data. Pattern Recog. (12) 165-175

Mőcsényi, M. (1968). A táj és a zöldterület fogalmi problémái a tájrendezés nézőpontjából. Településtudományi Közlemények. (21), 66-76

Nicol, S., Roach, J. K., Griffith, B. (2013) Spatial heterogeneity in statistical power to detect changes in lake area in Alaskan National Wildlife Refuges. Landscape Ecol. (28), 507-517 doi: 10.1007/s10980-013-9853-5

Riitters, K. H., Jones, K. B. (1996). A Landscape Atlas of the Chesapeake Bay Watershed. U.S. Environmental Protection Agency, Washington, DC, EPA/600/S-96/003

Safavian, S. R., Landgrebe, D. A. (1991). Survey of decision tree classifier methodology. Systems, Man and Cybernetics, IEEE Trans. (3) 660-674

Strohmeier, G. (2007). Werkmaterialen zur Landschaftswahrnehmung. Österreichisches Portal zur Umweltbildung und nachhaltigen Entwicklung URL: http://www.umweltbildung.at (12.02.2008)

Urban, D. L., O'Neill, R. V., Shugart, H. H. (1987). Landscape ecology. BioScience, 37/2:119-127

Woodcock, C. E., Strahler, A. H. (1987) The Factor of Scale in Remote Sensing. Remote Sensing of Environment (21), 311-332

$\mathrm{Wu}$, J. (2004). Effects of changing scale on landscape pattern analysis: scaling relations. Landscape Ecology (19), 125-138 